\title{
Characteristics and Clinical Outcomes of Children and Adolescents Aged $<18$ Years Hospitalized with COVID-19 - Six Hospitals, United States, July-August 2021
}

\author{
Valentine Wanga, $\mathrm{PhD}^{1,2}$; Megan E. Gerdes, MPH${ }^{1}$; Dallas S. Shi, MD, PhD ${ }^{1,2}$; Rewa Choudhary, MD ${ }^{1,2}$; Theresa M. Dulski, MD ${ }^{1,2}$; \\ Sophia Hsu, MSN, MPH ${ }^{1}$; Osatohamwen I. Idubor, MD ${ }^{1}$; Bryant J. Webber, MD ${ }^{1,2}$; Arthur M. Wendel, MD ${ }^{1}$; Nickolas T. Agathis, MD ${ }^{1,2}$; \\ Kristi Anderson, $\mathrm{MD}^{1}$; Tricia Boyles, MHA ${ }^{1}$; Sophia K. Chiu, MD ${ }^{1}$; Eleanor S. Click, MD, PhD ${ }^{1}$; Juliana Da Silva, MD ${ }^{1}$; Hannah Dupont, MPH ${ }^{1}$; \\ Mary Evans, MD ${ }^{1}$; Jeremy A.W. Gold, MD ${ }^{1}$; Julia Haston, MD ${ }^{1,2}$; Pamela Logan, MD ${ }^{1}$; Susan A. Maloney, MD ${ }^{1}$; Marisol Martinez, PharmD ${ }^{1}$; \\ Pavithra Natarajan, BMBS ${ }^{1}$; Kevin B. Spicer, MD, PhD ${ }^{1}$; Mark Swancutt, MD ${ }^{1}$; Valerie A. Stevens ${ }^{1}$; Jessica Brown, PhD ${ }^{1}$; Gyan Chandra, MBA ${ }^{1}$; \\ Megan Light, MPH ${ }^{1}$; Frederick E. Barr, MD 3 ; Jessica Snowden, MD 3 ; Larry K. Kociolek, MD ${ }^{4}$; Matthew McHugh, MPH ${ }^{4}$; David Wessel, MD 5 ; \\ Joelle N. Simpson, MD5; Kathleen C. Gorman, MSN5; Kristen A. Breslin, MD5; Roberta L. DeBiasi, MD ${ }^{5}$; Aaron Thompson, MD ${ }^{6,7}$; \\ Mark W. Kline, MD ${ }^{6,7}$; Julie A. Bloom, MD ${ }^{8}$; Ila R. Singh, MD, PhD ${ }^{9}$; Michael Dowlin ${ }^{9}$; Mark Wietecha, MS, MBA ${ }^{10}$; Beth Schweitzer, MS ${ }^{1}$; \\ Sapna Bamrah Morris, $\mathrm{MD}^{1}$; Emily H. Koumans, $\mathrm{MD}^{1}$; Jean Y. Ko, $\mathrm{PhD}^{1}$; Anne A. Kimball, MD ${ }^{1, *}$; David A. Siegel, MD ${ }^{1, *}$
}

During June 2021, the highly transmissible ${ }^{\dagger}$ B.1.617.2 (Delta) variant of SARS-CoV-2, the virus that causes COVID-19, became the predominant circulating strain in the United States. U.S. pediatric COVID-19-related hospitalizations increased during July-August 2021 following emergence of the Delta variant and peaked in September 2021..$^{\$}$ As of May 12, 2021, CDC recommended COVID-19 vaccinations for persons aged $\geq 12$ years, 9 and on November 2, 2021, COVID-19 vaccinations were recommended for persons aged 5-11 years.** To date, clinical signs and symptoms, illness course, and factors contributing to hospitalizations during the period of Delta predominance have not been well described in pediatric patients. CDC partnered with six children's hospitals to review medical record data for patients aged $<18$ years with COVID-19-related hospitalizations during July-August 2021. ${ }^{\dagger \dagger}$ Among 915 patients identified, 713 (77.9\%) were hospitalized for COVID-19 (acute COVID-19 as the primary or contributing reason for hospitalization), 177 (19.3\%) had incidental positive SARS-CoV-2 test results (asymptomatic or mild infection unrelated to the reason for hospitalization), and $25(2.7 \%)$ had multisystem inflammatory syndrome in children (MIS-C), a rare but serious inflammatory condition associated with COVID-19. $\$ \$$ Among the 713 patients

\footnotetext{
* These authors contributed equally to this report.

${ }^{\dagger}$ https://www.cdc.gov/coronavirus/2019-ncov/variants/delta-variant.html; https://covid.cdc.gov/covid-data-tracker/\#variant-proportions (Accessed September 15, 2021).

$\$$ https://covid.cdc.gov/covid-data-tracker/\#new-hospital-admissions

I https://www.cdc.gov/mmwr/volumes/70/wr/mm7020e1.htm?s_cid=mm7020e1_w

** https://www.cdc.gov/mmwr/volumes/70/wr/mm7045e1.htm?s_cid=mm7045e1_w

$\dagger \dagger$ COVID-19 was confirmed with laboratory detection of SARS-CoV-2 by reverse transcription-polymerase chain reaction or antigen test.

$\$ \$$ Patients with MIS-C as the reason for hospitalization included patients who met the clinical case definition for MIS-C (clinically severe illness requiring hospitalization in a person aged $<21$ years with fever, laboratory evidence of inflammation, multisystem [ $[22]$ organ involvement and no alternative plausible diagnosis, and evidence of current or recent SARS-CoV-2 infection by reverse transcription polymerase chain reaction, serology or antigen test, or COVID-19 exposure within the 4 weeks preceding symptom onset [https:// emergency.cdc.gov/han/2020/han00432.asp]) and were hospitalized for diagnosis and management of MIS-C, based on chart review.
}

hospitalized for COVID-19, 24.7\% were aged $<1$ year, $17.1 \%$ were aged $1-4$ years, $20.1 \%$ were aged $5-11$ years, and $38.1 \%$ were aged 12-17 years. Approximately two thirds of patients $(67.5 \%)$ had one or more underlying medical conditions, with obesity being the most common (32.4\%); among patients aged 12-17 years, $61.4 \%$ had obesity. Among patients hospitalized for COVID-19, 15.8\% had a viral coinfection'9 $(66.4 \%$ of whom had respiratory syncytial virus [RSV] infection). Approximately one third (33.9\%) of patients aged $<5$ years hospitalized for COVID-19 had a viral coinfection. Among 272 vaccine-eligible (aged 12-17 years) patients hospitalized for COVID-19, one $(0.4 \%)$ was fully vaccinated.*** Approximately one half $(54.0 \%)$ of patients hospitalized for COVID-19 received oxygen support, $29.5 \%$ were admitted to the intensive care unit (ICU), and 1.5\% died; of those requiring respiratory support, $14.5 \%$ required invasive mechanical ventilation (IMV). Among pediatric patients with COVID-19related hospitalizations, many had severe illness and viral coinfections, and few vaccine-eligible patients hospitalized for COVID-19 were vaccinated, highlighting the importance of vaccination for those aged $\geq 5$ years and other prevention strategies to protect children and adolescents from COVID-19, particularly those with underlying medical conditions.

\footnotetext{
99 Patients were considered to have a viral coinfection if they had $\geq 1$ of the following infections: type $A$ influenza, type $B$ influenza, unspecified influenza, coronavirus $229 \mathrm{e}$, coronavirus hku1, coronavirus nl63, coronavirus 0c43, respiratory syncytial virus, adenovirus, parainfluenza type 1 , parainfluenza type 2 , parainfluenza type 3 , parainfluenza type 4 , human metapneumovirus, rhinovirus, enterovirus, or other viral coinfection.

*** Fully vaccinated was defined as having received 2 doses of an mRNA-based COVID-19 vaccine $\geq 14$ days before hospital admission date. Partially vaccinated was defined as having received only 1 dose of an mRNA-based COVID-19 vaccine $\geq 14$ days before hospitalization. All vaccinated patients in this study received the Pfizer-BioNTech (BNT162b2) vaccine.
} 
Data were collected from six U.S. children's hospitals located in areas with high COVID-19 incidence during July-August 2021 (Arkansas, District of Columbia, Florida, Illinois, Louisiana, and Texas). ${ }^{\dagger \dagger}$ Data from hospitalized patients aged $<18$ years with COVID-19 or SARS-CoV-2 infection ${ }^{\$ S \$}$ were abstracted from electronic medical records using REDCap software (version 11.1.8; Vanderbilt University). Patients were categorized 999 by reason for hospitalization: 1) acute COVID-19, 2) incidental positive SARS-CoV-2 test result, or

\footnotetext{
${ }^{\dagger \dagger \dagger}$ A convenience sample of six hospitals was selected among members of the Children's Hospital Association. All hospitals were in jurisdictions with a high level of COVID-19 community transmission during July-August 2021; these jurisdictions were not represented by the COVID-NET surveillance system. https://www.cdc.gov/coronavirus/2019-ncov/covid-data/covid-net/ purpose-methods.html

S\$S COVID-19 diagnosis indicated in medical record or based on positive SARS-CoV-2 test result (antigen or polymerase chain reaction/nucleic acid amplification test, or antibody test among patients with a diagnosis of MIS-C).

999 Abstractors selected the category that best fit the overall reason for hospitalization, with adjudication by project leaders, and through audits of $5 \%$ of all charts.
}

3) MIS-C. Patient demographic characteristics, medical history, coinfections, and disease severity, including need for and duration of respiratory support, ICU admission, IMV, extracorporeal membrane oxygenation (ECMO), ,*** and deaths were abstracted from the medical record. Among patients hospitalized for COVID-19, presence of underlying medical conditions (including obesity), ${ }^{\dagger \dagger \dagger}$ viral coinfection, and illness course were described by age group. Pearson's chi-square and Kruskal-Wallis tests were used to compare categorical and continuous variables, respectively; $\mathrm{p}$-values $<0.05$ were

**** ECMO is a form of advanced life support used in patients with medically refractory respiratory or cardiac failure.

$\dagger \dagger \dagger$ For children aged $\geq 2$ years, height and weight were used to calculate body mass index (BMI) $\left(\mathrm{kg} / \mathrm{m}^{2}\right)$. BMI percentiles were calculated using BMI, age, and sex. Children with BMI percentiles $\geq 95 \%$ were considered to have obesity (https://www.cdc.gov/obesity/childhood/defining.html) and those with BMI $\geq 120 \%$ of the 95 th percentile were considered to have severe obesity. BMI data extracted from charts was used if height or weight was missing. If BMI was missing or unable to be calculated, a diagnosis of obesity recorded in charts was used and severity of obesity was unable to be assessed. Obesity was not assessed for children aged $<2$ years.

TABLE 1. Demographic characteristics and COVID-19 vaccination status of hospitalized patients aged <18 years with a positive SARS-CoV-2 test result or diagnosis of COVID-19, by reason for hospitalization - six hospitals, ${ }^{*}$ United States, July-August 2021

\begin{tabular}{|c|c|c|c|c|}
\hline \multirow[b]{3}{*}{ Characteristic } & \multicolumn{4}{|c|}{ Reason for hospitalization, no. (\%) } \\
\hline & Overall & COVID-19 ${ }^{\dagger}$ & Incidental positive SARS-CoV-2 test result & MIS-C§ \\
\hline & $N=915(100)$ & $\mathrm{n}=713(77.9)$ & $n=177(19.3)$ & $n=25(2.7)$ \\
\hline Age, yrs, median (IQR) & $8.0(1.3-14.0)$ & $8.0(1.0-14.0)$ & $9.0(2.0-14.0)$ & $8.0(4.0-13.0)$ \\
\hline \multicolumn{5}{|l|}{ Age group, yrs } \\
\hline$<1$ & $206(22.5)$ & $176(24.7)$ & $29(16.4)$ & $1(4.0)$ \\
\hline $1-4$ & $167(18.3)$ & $122(17.1)$ & $36(20.3)$ & $9(36.0)$ \\
\hline $5-11$ & $197(21.5)$ & $143(20.1)$ & $47(26.6)$ & $7(28.0)$ \\
\hline $12-17$ & $345(37.7)$ & $272(38.1)$ & $65(36.7)$ & $8(32.0)$ \\
\hline \multicolumn{5}{|l|}{ Sex } \\
\hline Female & $437(47.8)$ & $340(47.7)$ & $87(49.2)$ & $10(40.0)$ \\
\hline Male & $478(52.2)$ & $373(52.3)$ & $90(50.8)$ & $15(60.0)$ \\
\hline \multicolumn{5}{|l|}{ Race/Ethnicity } \\
\hline White, non-Hispanic & $277(30.3)$ & $210(29.5)$ & $59(33.3)$ & $8(32.0)$ \\
\hline Black or African American, non-Hispanic & $260(28.4)$ & $202(28.3)$ & $48(27.1)$ & $10(40.0)$ \\
\hline Hispanic & $267(29.2)$ & $211(29.6)$ & $52(29.4)$ & $4(16.0)$ \\
\hline Other, Non-Hispanic" & $42(4.6)$ & $35(4.9)$ & $6(3.4)$ & $1(4.0)$ \\
\hline Unknown & $69(7.5)$ & $55(7.7)$ & $12(6.8)$ & $2(8.0)$ \\
\hline \multicolumn{5}{|l|}{ COVID-19 vaccination status } \\
\hline Eligible for vaccination (aged 12-17 yrs)** & $345(37.7)$ & $272(38.1)$ & $65(36.7)$ & $8(32.0)$ \\
\hline Fully vaccinated & $3(0.9)$ & $1(0.4)$ & $2(3.1)$ & $0(-)$ \\
\hline Partially vaccinated & $18(5.2)$ & $12(4.4)$ & $4(6.2)$ & $2(25.0)$ \\
\hline Not vaccinated & $224(64.9)$ & $196(72.1)$ & $22(33.8)$ & $6(75.0)$ \\
\hline Unknown vaccination status & $100(29.0)$ & $63(23.2)$ & 37 (56.9) & $0(-)$ \\
\hline Ineligible for vaccination (aged $<12 \mathrm{yrs}$ ) & $570(62.3)$ & $441(61.9)$ & $112(63.3)$ & $17(68.0)$ \\
\hline
\end{tabular}

Abbreviation: MIS-C = multisystem inflammatory syndrome in children.

* The six children's hospitals were in Arkansas, District of Columbia, Florida, Illinois, Louisiana, and Texas.

† Patients hospitalized for COVID-19 included patients with acute COVID-19 as the primary reason for hospitalization or with acute COVID-19 as a secondary or contributing reason for hospitalization, based on chart review.

$\S$ Patients with MIS-C as the reason for hospitalization included patients who met the clinical case definition for MIS-C (clinically severe illness requiring hospitalization in a person aged $<21$ years with fever, laboratory evidence of inflammation, multisystem [ $\geq 2]$ organ involvement and no alternative plausible diagnosis, and evidence of current or recent SARS-CoV-2 infection by reverse transcription polymerase chain reaction, serology or antigen test, or COVID-19 exposure within the 4 weeks preceding symptom onset [https://emergency.cdc.gov/han/2020/han00432.asp]) and were hospitalized for diagnosis and management of MIS-C, based on chart review.

I Other race/ethnicity includes Asian, Native Hawaiian or Other Pacific Islander, American Indian or Alaska Native, and Other (not specified).

** Fully vaccinated was defined as having received 2 doses of an mRNA-based COVID-19 vaccine $\geq 14$ days before the hospital admission date. Partially vaccinated was defined as having received only 1 dose of an mRNA-based COVID-19 vaccine $\geq 14$ days before hospitalization. All vaccinated patients in this study received the Pfizer-BioNTech (BNT162b2) vaccine. 
considered statistically significant. All analyses were conducted using SAS (version 9.4; SAS Institute) and R (Version 4.0.3; $\mathrm{R}$ Foundation for Statistical Computing). This activity was reviewed by CDC and the other participating institutions and was conducted consistent with applicable federal law and CDC policy. $\$ \$ \$ \$ \$$

\$s\$\$ 45 C.F.R. part 46, 21 C.F.R. part 56; 42 U.S.C. Sect. 241(d); 5 U.S.C. Sect. 552a; 44 U.S.C. Sect. 3501 et seq.
Among 915 patients aged $<18$ years, 713 (77.9\%) were hospitalized for COVID-19, 177 (19.3\%) had incidental SARS-CoV-2 infections, and 25 (2.7\%) had MIS-C (Table 1). Among all 915 patients, $22.5 \%$ were aged $<1$ year, $18.3 \%$ were aged $1-4$ years, $21.5 \%$ were aged $5-11$ years, and $37.7 \%$ were aged 12-17 years. Among the 713 patients hospitalized for COVID-19, approximately one half $(373 ; 52.3 \%)$ were male, $210(29.5 \%)$ were non-Hispanic White persons, 202 (28.3\%)

TABLE 2. Hospitalization and illness course among children and adolescents aged <18 years hospitalized for COVID-19,* by age group six hospitals, ${ }^{\dagger}$ United States, July-August 2021

\begin{tabular}{|c|c|c|c|c|c|c|}
\hline \multirow[b]{3}{*}{ Characteristic } & \multicolumn{6}{|c|}{ Age group, yrs, no. (\%) } \\
\hline & Overall & $<1$ & $1-4$ & $5-11$ & $12-17$ & \\
\hline & $(\mathrm{N}=713)$ & $(n=176)$ & $(n=122)$ & $(n=143)$ & $(n=272)$ & p-value \\
\hline \multicolumn{7}{|l|}{ No. of underlying medical conditions } \\
\hline None & $232(32.5)$ & $124(70.5)$ & $51(41.8)$ & $25(17.5)$ & $32(11.8)$ & $<0.001$ \\
\hline $1-2$ & $366(51.3)$ & $47(26.7)$ & $46(37.7)$ & $89(62.2)$ & $184(67.6)$ & \\
\hline$\geq 3$ & $115(16.1)$ & $5(2.8)$ & $25(20.5)$ & $29(20.3)$ & $56(20.6)$ & \\
\hline \multicolumn{7}{|l|}{ Five most prevalent conditions by system } \\
\hline Metabolic or endocrine? & $258(36.2)$ & $2(1.1)$ & $17(13.9)$ & $59(41.3)$ & $180(66.2)$ & $<0.001$ \\
\hline Obesity** & $231(32.4)$ & - & $16(13.1)$ & $48(33.6)$ & $167(61.4)$ & \\
\hline Obesity & $90(39.0)$ & - & $14(87.5)$ & $17(35.4)$ & $59(35.3)$ & $<0.001$ \\
\hline Severe obesity & $131(56.7)$ & - & $1(6.3)$ & $29(60.4)$ & $101(60.5)$ & \\
\hline Obesity, unknown severity & $10(4.3)$ & - & $1(6.3)$ & $2(4.2)$ & $7(4.2)$ & \\
\hline Neurologic or developmental ${ }^{\dagger \dagger}$ & $144(20.2)$ & $41(23.3)$ & $33(27.0)$ & $28(19.6)$ & $42(15.4)$ & 0.038 \\
\hline Seizure disorder & $57(8.0)$ & $6(3.4)$ & $15(12.3)$ & $14(9.8)$ & $22(8.1)$ & 0.033 \\
\hline Respiratory§§ & $142(19.9)$ & $7(4.0)$ & $18(14.8)$ & $34(23.8)$ & $83(30.5)$ & $<0.001$ \\
\hline Asthma or RAD & $114(16.0)$ & $2(1.1)$ & $12(9.8)$ & $26(18.2)$ & $74(27.2)$ & $<0.001$ \\
\hline Gastrointestinal or hepatic & $85(11.9)$ & $12(6.8)$ & $28(23.0)$ & $16(11.2)$ & 29 (10.7) & $<0.001$ \\
\hline Feeding tube dependent & $59(8.3)$ & $7(4.0)$ & $23(18.9)$ & $13(9.1)$ & $16(5.9)$ & $<0.001$ \\
\hline Psychiatric $* * *$ & $58(8.1)$ & $0(-)$ & $0(-)$ & $13(9.1)$ & 45 (16.5) & $<0.001$ \\
\hline Depression & $23(3.2)$ & $0(-)$ & $0(-)$ & $1(0.7)$ & $22(8.1)$ & $<0.001$ \\
\hline \multicolumn{7}{|l|}{ Multiple admissions } \\
\hline Yes & $28(3.9)$ & $4(2.3)$ & $5(4.1)$ & $6(4.2)$ & $13(4.8)$ & 0.607 \\
\hline No & $685(96.1)$ & $172(97.7)$ & $117(95.9)$ & $137(95.8)$ & $259(95.2)$ & \\
\hline Unknown & $14(2.4)$ & $3(1.9)$ & $2(2.0)$ & $2(1.9)$ & $6(2.7)$ & \\
\hline Hospital length of stay, median days (IQR) & $3.0(1.0-7.0)$ & $3.0(1.0-6.8)$ & $3.0(2.0-4.5)$ & $3.0(1.0-7.0)$ & $4.0(2.0-8.0)$ & 0.187 \\
\hline \multicolumn{7}{|l|}{ Admitted to ICU } \\
\hline Yes & $210(29.5)$ & $34(19.3)$ & $31(25.4)$ & 37 (25.9) & $108(39.7)$ & $<0.001$ \\
\hline No & $503(70.5)$ & $142(80.7)$ & 91 (74.6) & $106(74.1)$ & $164(60.3)$ & \\
\hline Total length of stay in ICU, median days (IQR) & $3.0(1.0-7.0)$ & $3.0(1.0-6.8)$ & $3.0(2.0-4.5)$ & $3.0(1.0-7.0)$ & $4.0(2.0-8.0)$ & 0.187 \\
\hline \multicolumn{7}{|l|}{ Highest level of respiratory support required } \\
\hline No oxygen support & $328(46.0)$ & $94(53.4)$ & $57(46.7)$ & $82(57.3)$ & $95(34.9)$ & $<0.001$ \\
\hline Oxygen support & $385(54.0)$ & $82(46.6)$ & $65(53.3)$ & $61(42.7)$ & $177(65.1)$ & \\
\hline Nasal cannula & $111(28.8)$ & $22(26.8)$ & $14(21.5)$ & $24(39.3)$ & $51(28.8)$ & \\
\hline Mask & $7(1.8)$ & $0(-)$ & $2(3.1)$ & $1(1.6)$ & $4(2.3)$ & \\
\hline CPAP or BiPAP & 69 (17.9) & $5(6.1)$ & $10(15.4)$ & $11(18.0)$ & $43(24.3)$ & \\
\hline High-flow nasal cannula & $142(36.9)$ & $43(52.4)$ & $32(49.2)$ & $14(23.0)$ & $53(29.9)$ & \\
\hline IMV & $56(14.5)$ & $12(14.6)$ & $7(10.8)$ & $11(18.0)$ & 26 (14.7) & \\
\hline Duration on IMV, median days (IQR) & $7.0(4.0-14.0)$ & $6.0(4.8-12.3)$ & $6.0(2.0-11.5)$ & $5.5(1.8-10.3)$ & $9.5(5.0-21.3)$ & 0.596 \\
\hline \multicolumn{7}{|l|}{ ECMO required } \\
\hline Yes & $8(1.1)$ & $1(0.6)$ & $1(0.8)$ & $1(0.7)$ & $5(1.8)$ & 0.567 \\
\hline No & 705 (98.9) & 175 (99.4) & $121(99.2)$ & $142(99.3)$ & $267(98.2)$ & \\
\hline Duration on ECMO, median days (IQR) & $12.0(5.5-17.8)$ & $1.0(1.0-1.0)$ & $13.0(13.0-13.0)$ & - & $15.0(11.0-26.0)$ & 0.247 \\
\hline Viral coinfection ${ }^{++\dagger}$ & $113(15.8)$ & $57(32.4)$ & $44(36.1)$ & $6(4.2)$ & $6(2.2)$ & $<0.001$ \\
\hline RSV & $75(66.4)$ & $42(73.7)$ & $26(59.1)$ & $4(66.7)$ & $3(50.0)$ & $<0.001$ \\
\hline \multicolumn{7}{|l|}{ Discharge status } \\
\hline Discharged alive & 702 (98.5) & 174 (98.9) & $122(100.0)$ & $142(99.3)$ & $264(97.1)$ & 0.231 \\
\hline Deceased & $11(1.5)$ & $2(1.1)$ & $0(-)$ & $1(0.7)$ & $8(2.9)$ & \\
\hline
\end{tabular}

See table footnotes on the next page. 
TABLE 2. (Continued) Hospitalization and illness course among children and adolescents aged <18 years hospitalized for COVID-19, ${ }^{*}$ by age group - six hospitals, ${ }^{\dagger}$ United States, July-August 2021

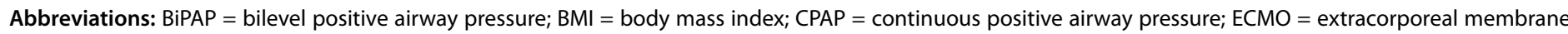
oxygenation; ICU = intensive care unit; IMV = invasive mechanical ventilation; RAD = reactive airway disease; RSV = respiratory syncytial virus.

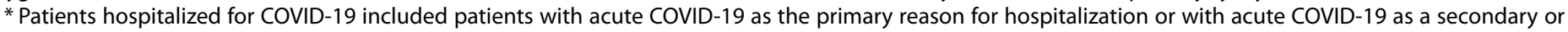
contributing reason for hospitalization, based on chart review.

† The six children's hospitals were in Arkansas, District of Columbia, Florida, Illinois, Louisiana, and Texas.

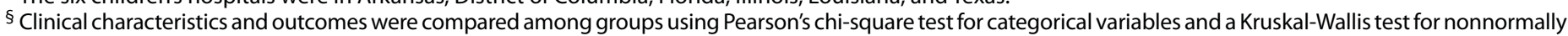
distributed variables.

" Metabolic and endocrine conditions included dyslipidemia, obesity, thyroid disorder, type 1 diabetes, type 2 diabetes, and other endocrine disorders.

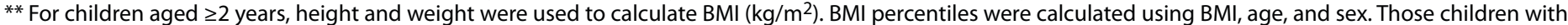

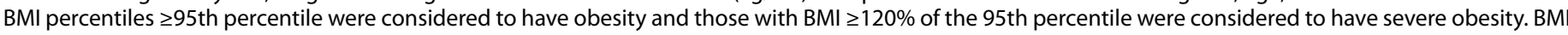

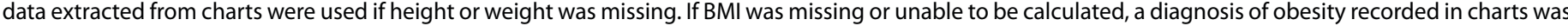
used and severity of obesity was unable to be assessed. Obesity was not assessed for children aged $<2$ years.

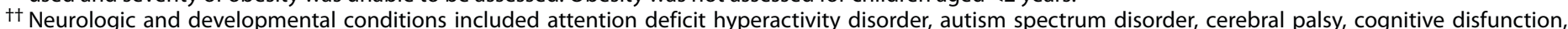

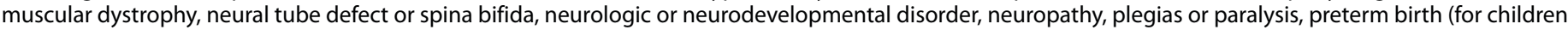
aged $<2$ years only), seizure disorder, and wheelchair/walker-dependence or bed-bound status.

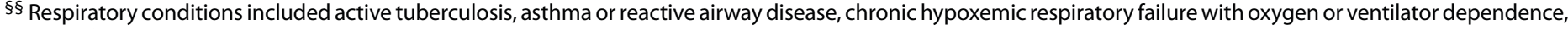
cystic fibrosis, current smoking or e-cigarette use, tracheostomy dependence, and other chronic lung diseases.

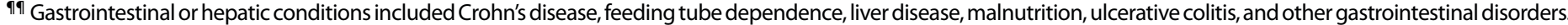

*** Psychiatric conditions included anxiety, borderline personality disorder, depression, substance use disorder, and other psychiatric diagnoses.

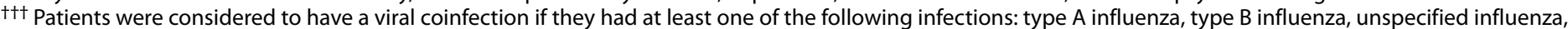

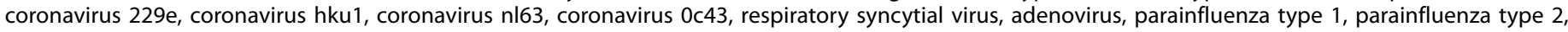
parainfluenza type 3, parainfluenza type 4, human metapneumovirus, rhinovirus enterovirus, or other viral coinfection.

were non-Hispanic Black persons or African American persons (Black), and 211 (29.6\%) were Hispanic persons.

Among the 713 patients hospitalized for COVID-19, $32.5 \%, 51.3 \%$, and $16.1 \%$ had zero, one or two, and three or more underlying medical conditions, respectively (Table 2). The most common conditions were obesity (32.4\%), asthma or reactive airway disease $(16.0 \%)$, and feeding tube dependence (8.3\%). Among patients aged $12-17$ years, $61.4 \%$ had obesity (60.5\% of whom had severe obesity). Among patients aged $5-11$ years, $33.6 \%$ had obesity $(60.4 \%$ of whom had severe obesity). Among patients hospitalized for COVID-19, 210 (29.5\%) had ICU admissions, eight (1.1\%) received ECMO, and $11(1.5 \%)$ died. Of the $385(54.0 \%)$ patients hospitalized for COVID-19 who received oxygen support, high-flow nasal cannula was the most common highest level of support (142; 36.9\%); 56 (14.5\%) patients received IMV. Across all age groups, the median hospital stay was 3 days, and the median IMV duration was 7 days. Patients aged 12-17 years had the longest median hospitalizations ( 4 days) and IMV requirement (9.5 days). Viral coinfection was common among patients aged $<1$ year $(32.4 \%)$ and $1-4$ years $(36.1 \%)$; overall, approximately two thirds of viral coinfections were with RSV (Table 2).

Among 272 vaccine-eligible patients hospitalized for COVID-19, one (0.4\%) was fully vaccinated and $12(4.4 \%)$ were partially vaccinated with an mRNA COVID-19 vaccine at the time of hospitalization (Table 1).

A higher percentage of patients hospitalized for COVID-19 with any underlying condition were admitted to the ICU (34.7\%) compared with those without an underlying condition $(18.5 \%)(p<0.001)$ (Table 3$)$. The duration of hospitalization was longer for patients with obesity (median $=4$ days
$[\mathrm{IQR}=2.0-7.5$ days $])$ than that for those without obesity (median $=2$ days $[\mathrm{IQR}=1.0-5.0$ days $])(\mathrm{p}<0.001)$. A higher proportion of patients with obesity were admitted to the ICU $(41.1 \%)$ than were those without obesity $(23.9 \%)(\mathrm{p}<0.001)$. A higher proportion of patients with viral coinfection required oxygen support $(69.0 \%)$ compared with those without viral coinfection $(51.2 \%)(\mathrm{p}<0.001)$.

\section{Discussion}

In this study of six U.S. hospitals during July-August, 2021, approximately three quarters of pediatric patients with COVID-19-related hospitalizations were hospitalized for COVID-19. The majority of those hospitalized for COVID-19 were Black or Hispanic and were aged $<5$ or 12-17 years. Approximately one third of patients aged $<1$ and 1-4 years had a viral coinfection, approximately one third of patients aged 5-11 years and approximately two thirds of patients aged 12-17 years had obesity. Less than $1 \%$ of vaccineeligible patients were fully vaccinated against COVID-19.

Five of the six hospitals had policies to test all pediatric patients for SARS-CoV-2 upon admission during the study period, allowing for detection of incidental positive SARS-CoV-2 test results. However, the proportion of such patients was smaller in this study compared with that in a previous report (1). Patients aged $0-4$ and 12-17 years accounted for 79\% of COVID-19-related hospitalizations in this study, which is consistent with data from other hospitals and communities (2). Among hospitalized children aged $<5$ years, most were aged $<1$ year, which might reflect clinical practice differences, because infants might be more likely to be hospitalized with milder disease than older children (3). Most patients were 
TABLE 3. Hospitalization and illness course among children and adolescents aged $<18$ years hospitalized for COVID-19* by presence of underlying medical conditions, obesity, and viral coinfection — six hospitals, ${ }^{\dagger}$ United States, July-August 2021

\begin{tabular}{|c|c|c|c|c|c|c|c|c|c|}
\hline \multirow[b]{3}{*}{ Characteristic } & \multicolumn{9}{|c|}{ No. (\%) } \\
\hline & \multicolumn{3}{|c|}{ Underlying medical condition } & \multicolumn{3}{|c|}{ Obesity§ } & \multicolumn{3}{|c|}{ Viral coinfection } \\
\hline & Yes $(n=481)$ & No $(n=232)$ & p-value & Yes $(n=231)$ & No $(n=482)$ & p-value q $^{2}$ & Yes $(n=113)$ & No $(n=600)$ & p-value \\
\hline \multicolumn{10}{|l|}{ Multiple admissions } \\
\hline Yes & $23(4.8)$ & $5(2.2)$ & 0.137 & $12(5.2)$ & $16(3.3)$ & 0.317 & $3(2.7)$ & $25(4.2)$ & 0.621 \\
\hline No & $458(95.2)$ & $227(97.8)$ & & $219(94.8)$ & $466(96.7)$ & & $110(97.3)$ & $575(95.8)$ & \\
\hline $\begin{array}{l}\text { Hospital length of stay, } \\
\text { median days (IQR) }\end{array}$ & $3.0(2.0-7.0)$ & $2.0(1.0-4.0)$ & $<0.001$ & $4.0(2.0-7.5)$ & $2.0(1.0-5.0)$ & $<0.001$ & $3.0(2.0-6.0)$ & $3.0(1.0-6.0)$ & 0.085 \\
\hline \multicolumn{10}{|l|}{ Admitted to ICU } \\
\hline Yes & $167(34.7)$ & $43(18.5)$ & $<0.001$ & $95(41.1)$ & $115(23.9)$ & $<0.001$ & $36(31.9)$ & $174(29.0)$ & 0.618 \\
\hline No & $314(65.3)$ & $189(81.5)$ & & $136(58.9)$ & $367(76.1)$ & & $77(68.1)$ & $426(71.0)$ & \\
\hline $\begin{array}{l}\text { ICU length of stay, } \\
\text { median days (IQR) }\end{array}$ & $4.0(1.0-8.0)$ & $2.0(1.0-4.0)$ & 0.023 & $4.0(2.0-8.0)$ & $3.0(1.0-6.5)$ & 0.014 & $4.0(1.8-10.3)$ & $3.0(1.0-7.0)$ & 0.37 \\
\hline \multicolumn{10}{|c|}{ Highest level of respiratory support required } \\
\hline None & $199(41.4)$ & $129(55.6)$ & $<0.001$ & $61(26.4)$ & $267(55.4)$ & $<0.001$ & $35(31.0)$ & $293(48.8)$ & $<0.001$ \\
\hline Oxygen support & $282(58.6)$ & $103(44.4)$ & & $170(73.6)$ & $215(44.6)$ & & $78(69.0)$ & 307 (51.2) & \\
\hline Nasal cannula & $77(27.3)$ & $34(33.0)$ & & $47(27.6)$ & $64(29.8)$ & & $10(12.8)$ & $101(32.9)$ & \\
\hline Mask & $6(2.1)$ & $1(1.0)$ & & $2(1.2)$ & $5(2.3)$ & & $1(1.3)$ & $6(2.0)$ & \\
\hline CPAP or BIPAP & $62(22.0)$ & $7(6.8)$ & & $43(25.3)$ & $26(12.1)$ & & $8(10.3)$ & $61(20.0)$ & \\
\hline High-flow nasal cannula & $91(32.3)$ & $51(49.5)$ & & $55(32.4)$ & $87(40.5)$ & & $46(59.0)$ & $96(31.3)$ & \\
\hline IMV & $46(16.3)$ & $10(9.7)$ & & $23(13.5)$ & $33(15.3)$ & & 13 (16.7) & $43(14.0)$ & \\
\hline $\begin{array}{l}\text { IMV duration, median } \\
\text { days (IQR) }\end{array}$ & $8.0(4.0-15.0)$ & $5.5(1.0-6.8)$ & 0.161 & $8.0(5.0-14.5)$ & $6.0(3.8-13.5)$ & 0.472 & $6.0(5.0-13.0)$ & $7.0(3.0-14.5)$ & 0.804 \\
\hline \multicolumn{10}{|l|}{ ECMO required } \\
\hline Yes & $5(1.0)$ & $3(1.3)$ & 1.000 & $5(2.2)$ & $3(0.6)$ & 0.147 & $2(1.8)$ & $6(1.0)$ & 0.821 \\
\hline No & $476(99.0)$ & $229(98.7)$ & & $226(97.8)$ & $479(99.4)$ & & $111(98.2)$ & $594(99.0)$ & \\
\hline $\begin{array}{l}\text { ECMO duration, median } \\
\text { days (IQR) }\end{array}$ & $15.0(11.0-26.0)$ & $1.0(0.5-7.0)$ & 0.101 & $15.0(11.0-26.0)$ & $1.0(0.5-7.0)$ & 0.101 & $7.0(4.0-10.0)$ & $13.0(8.0-23.3)$ & 0.505 \\
\hline Viral coinfection** & $49(10.2)$ & $64(27.6)$ & $<0.001$ & $7(3.0)$ & $106(22.0)$ & $<0.001$ & $113(100.0)$ & $0(-)$ & $<0.001$ \\
\hline RSV & $31(63.3)$ & 44 (68.8) & $<0.001$ & $2(28.6)$ & 73 (68.9) & $<0.001$ & 75 (66.4) & $0(-)$ & $<0.001$ \\
\hline \multicolumn{10}{|l|}{ Discharge status } \\
\hline Discharged alive & $472(98.1)$ & $230(99.1)$ & 0.517 & $227(98.3)$ & 475 (98.5) & 0.595 & $111(98.2)$ & $591(98.5)$ & 0.81 \\
\hline Deceased & $9(1.9)$ & $2(0.9)$ & & $4(1.7)$ & $7(1.5)$ & & $2(1.8)$ & $9(1.5)$ & \\
\hline
\end{tabular}

Abbreviations: BIPAP = bilevel positive airway pressure; $\mathrm{BMI}=$ body mass index; $\mathrm{CPAP}=$ continuous positive airway pressure; $\mathrm{ECMO}=$ extracorporeal membrane oxygenation; ICU = intensive care unit; IMV = invasive mechanical ventilation; RSV = respiratory syncytial virus.

* Patients hospitalized for COVID-19 included patients with acute COVID-19 as the primary reason for hospitalization or with acute COVID-19 as a secondary or contributing reason for hospitalization, based on chart review.

† The six children's hospitals were in Arkansas, District of Columbia, Florida, Illinois, Louisiana, and Texas.

$\S$ For children aged $\geq 2$ years, height and weight were used to calculate BMI $\left(\mathrm{kg} / \mathrm{m}^{2}\right)$. BMI percentiles were calculated using BMI, age, and sex. Those children with $\mathrm{BMI}$ percentiles $\geq 95$ th percentile were considered to have obesity, and those with $\mathrm{BMI} \geq 120 \%$ of the 95 th percentile were considered to have severe obesity. BMI data extracted from charts were used if height or weight was missing. If BMI was missing or unable to be calculated, a diagnosis of obesity recorded in charts was used and severity of obesity was unable to be assessed. Obesity was not assessed for children aged $<2$ years.

q Clinical characteristics and outcomes were compared among groups using Pearson's chi-square test for categorical variables and a Kruskal-Wallis test for nonnormally distributed variables.

** Patients were considered to have a viral coinfection if they had $\geq 1$ of the following infections: type A influenza, type B influenza, unspecified influenza, coronavirus 229 , coronavirus hku1, coronavirus nl63, coronavirus 0c43, respiratory syncytial virus, adenovirus, parainfluenza type 1, parainfluenza type 2, parainfluenza type 3 , parainfluenza type 4, human metapneumovirus, rhinovirus, enterovirus, or other viral coinfection.

Black or Hispanic in this study; an earlier study demonstrated higher hospitalization rates among Black or Hispanic children compared with White children (1).

Approximately two thirds of patients hospitalized for COVID-19, including $83 \%$ and $88 \%$ of patients aged $5-11$ and 12-17 years, respectively, had one or more underlying medical conditions. Approximately two thirds of patients hospitalized for COVID-19 aged 12-17 years had obesity. Compared with patients without obesity, those with obesity required higher levels and longer duration of care. These findings are consistent with previous reports (4) and highlight the importance of obesity and other medical conditions as risk factors for severe COVID-19 in children and adolescents.

The proportions of patients admitted to ICU and who required IMV are similar to those in prior reports, which predominantly included hospitalized pediatric COVID-19 patients before Delta variant predominance $(2,5)$. Adolescents were more likely to require ICU admission and oxygen support compared with other age groups and required the longest median duration of IMV. The median duration of IMV overall (7 days) is consistent with previous reports $(6,7)$. Approximately one half of patients aged $1-4$ years required 


\section{Summary}

What is already known about this topic?

Pediatric COVID-19-related hospitalization rates increased when the highly transmissible SARS-CoV-2 B.1.617.2 (Delta) variant became the predominant circulating strain.

What is added by this report?

Among children and adolescents with SARS-CoV-2 infection admitted to six hospitals during July-August 2021, 77.9\% were hospitalized for acute COVID-19. Among these patients, approximately one third aged $<5$ years had a viral coinfection (approximately two thirds of which were respiratory syncytial virus) and approximately two thirds of those aged 12-17 years had obesity; only $0.4 \%$ of age-eligible patients were fully vaccinated.

What are the implications for public health practice?

COVID-19 vaccination and other prevention strategies are important to protect children from COVID-19, particularly children with obesity and other underlying health conditions.

oxygen support, which might be related to the high proportion with viral coinfection. This study occurred during July-August 2021, the first period during the COVID-19 pandemic with high circulation of RSV9999 and other respiratory viruses. Compared with prior studies $(2,5)$, this study found a high proportion of patients had high-flow nasal cannula as the highest level of respiratory support (37\%), which might reflect a change in practice to avoid intubation or the high proportion of viral coinfections, including RSV.

On November 2, 2021, CDC recommended COVID-19 vaccinations for children aged 5-11 years (8). As of July 31, $2021,29 \%$ of U.S. persons aged $12-17$ years were fully vaccinated against COVID-19. ${ }^{* * * * *}$ In this study, only $0.4 \%$ of vaccine-eligible adolescents hospitalized for COVID-19 were fully vaccinated. Hospitalization rates have been shown to be 10 times higher among unvaccinated adolescents compared with fully vaccinated adolescents (2). Similarly, this study demonstrates that unvaccinated children hospitalized for COVID-19 could experience severe disease and reinforces the importance of vaccination of all eligible children to provide individual protection and to protect those who are not yet eligible to be vaccinated.

The findings in this report are subject to at least five limitations. First, the data came from only six hospitals, five of which are in the southern U.S. region. The proportion of adolescents with obesity in the southern United States is higher than in other regions, ${ }^{\dagger \dagger \dagger \dagger}$ which might explain the high rates

\footnotetext{
S999 https://emergency.cdc.gov/han/2021/han00443.asp; https://www.cdc.gov/ surveillance/nrevss/rsv/natl-trend.html

***** https://covid.cdc.gov/covid-data-tracker/\#vaccination-demographics-trends

$\dagger+\dagger \dagger$ https://nccd.cdc.gov/dnpao_dtm/rdPage.aspx?rdReport=DNPAO_DTM. ExploreByTopic\&islClass $=$ OWS $\&$ islTopic $=\& g o=G O$
}

of obesity described in this report. Therefore, findings might not be generalizable to other areas. Second, findings might reflect differences in practices by hospitals or changes in practice over time and might not reflect differences in severity of COVID-19 related to the Delta variant. Third, incomplete or missing data in medical records might lead to underreporting and underestimation of details such as COVID-19 vaccination frequencies. Fourth, at the time of hospitalization, persons aged $12-15$ years had only been vaccine-eligible for $2-3$ months (9), possibly contributing to the low vaccination rates observed. Finally, hospitals identified patients for review based on positive polymerase chain reaction and antigen SARS-CoV-2 test results and hospitalization during the study period. Therefore, proportions of patients with MIS-C are likely underestimated.

Among pediatric patients with COVID-19-related hospitalizations, many had severe illness and viral coinfections, and few vaccine-eligible patients hospitalized for COVID-19 were vaccinated. These data highlight the importance of COVID-19 vaccination for those aged $\geq 5$ years and other prevention strategies to protect children and adolescents from COVID-19, particularly those with obesity and other underlying health conditions. Further research and surveillance for viral coinfections with SARS-CoV-2 in pediatric patients can inform public health and capacity planning (10).

\section{Acknowledgments}

Anthony Bastiand, Troy Bienemy, Jerry Bridgham, Joyce Dalton, Laura Fisher, Barret Flagg, Jennifer Giovanni, Kara Hollis, Ashok Kurian, Brendan Jackson, Amy Knight, Veena Nagarajan, Aimee Ossman, Emily Paganelli, Georgina Peacock, Nicole Pereira-Abara, Trescena Preacher, Andrea Romaniuk, Leila Sahni, Susan Stark, Sherry Sweek, Daniella Van Der Merwe, James Versalovic.

Corresponding author: Valentine Wanga, qdy0@cdc.gov.

${ }^{1}$ CDC COVID-19 Response Team; ${ }^{2}$ Epidemic Intelligence Service, CDC; ${ }^{3}$ Arkansas Children's, Little Rock, Arkansas; ${ }^{4}$ Ann \& Robert H. Lurie Children's Hospital of Chicago, Chicago, Illinois; ${ }^{5}$ Children's National Hospital, Washington, DC; ${ }^{6}$ Children's Hospital New Orleans, New Orleans, Louisiana; ${ }^{7}$ Tulane University School of Medicine and LSU Health, New Orleans, Louisiana; ${ }^{8}$ Department of Pediatrics, Baylor College of Medicine, Houston, Texas; ${ }^{9}$ Department of Pathology and Immunology, Baylor College of Medicine, Houston, Texas; ${ }^{10}$ Children's Hospital Association, Washington, DC.

All authors have completed and submitted the International Committee of Medical Journal Editors form for disclosure of potential conflicts of interest. Ila R. Singh reports funding from the National Institutes of Health (NIH) as a coinvestigator for grant no. R61HD105593 to characterize pediatric COVID-19. Roberta L. DeBiasi reports grant support and contracts for COVID-19 and MIS-C, unrelated to the current work; consulting fees from I-ACT for Children; honoraria from the Infectious Diseases in Children Conference (NYC) and Children's Hospital Colorado Infectious Diseases Conference (Denver); and unpaid membership on the board of the Pediatric Infectious Diseases Society. Larry K. Kociolek 
reports a grant from the Walder Foundation Chicago Coronavirus Assessment Network Initiative, institutional support from Merck and NIH/NIAID; and honoraria for educational events at Northwest Community Hospital and Nemours/duPont Children's Hospital. Jessica Snowden reports institutional support from NIH Office of the Director-ECHO program and NIH/NHLBI-RECOVER program, unrelated to the current work. Frederick E. Barr reports application of patent 17364280 with Asklepion Pharmaceuticals for L-citrulline to prevent or treat endothelial dysfunction. Sapna Bamrah Morris and Sophia K. Chiu report membership on a data safety monitoring board in a study of ivermectin for treatment of severe COVID-19 in Ghana. Sophia Hsu reports ownership of 5 shares of Moderna stock and 7 shares of Novavax stock, and ownership within the past 36 months (but no current ownership) of stock in BioNTech, Gilead Sciences, and Pfizer. Theresa M. Dulski reports that her husband receives restricted stock units as part of his compensation from his employer, a cancer diagnostics company that also performs COVID-19 testing. No other potential conflicts of interest were disclosed.

\section{References}

1. Woodruff RC, Campbell AP, Taylor CA, et al.; COVID-NET surveillance team. Risk factors for severe COVID-19 in children. Pediatrics 2021;e2021053418. PMID:34686570 https://doi. org/10.1542/peds.2021-053418

2. Delahoy MJ, Ujamaa D, Whitaker M, et al.; COVID-NET Surveillance Team. Hospitalizations associated with COVID-19 among children and adolescents-COVID-NET, 14 States, March 1, 2020-August 14, 2021. MMWR Morb Mortal Wkly Rep 2021;70:1255-60. PMID:34499627 https://doi.org/10.15585/mmwr.mm7036e2

3. Antoon JW, Grijalva CG, Thurm C, et al. Factors associated with COVID-19 disease severity in US children and adolescents. J Hosp Med 2021;16:603-10. PMID:34613896 https://doi.org/10.12788/jhm.3689
4. Kompaniyets L, Agathis NT, Nelson JM, et al. Underlying medical conditions associated with severe COVID-19 illness among children. JAMA Netw Open 2021;4:e2111182. PMID:34097050 https://doi. org/10.1001/jamanetworkopen.2021.11182

5. Fernandes DM, Oliveira CR, Guerguis S, et al.; Tri-State Pediatric COVID-19 Research Consortium. Severe acute respiratory syndrome coronavirus 2 clinical syndromes and predictors of disease severity in hospitalized children and youth. J Pediatr 2021;230:23-31.e10. PMID:33197493 https://doi.org/10.1016/j.jpeds.2020.11.016

6. Newth CJ, Venkataraman S, Willson DF, et al.; Eunice Shriver Kennedy National Institute of Child Health and Human Development Collaborative Pediatric Critical Care Research Network. Weaning and extubation readiness in pediatric patients. Pediatr Crit Care Med 2009;10:1-11. PMID:19057432 https://doi.org/10.1097/ PCC.0b013e318193724d

7. Chao JY, Derespina KR, Herold BC, et al. Clinical characteristics and outcomes of hospitalized and critically ill children and adolescents with coronavirus disease 2019 at a tertiary care medical center in New York City. J Pediatr 2020;223:14-19.e2. PMID:32407719 https://doi. org/10.1016/j.jpeds.2020.05.006

8. Woodworth KR, Moulia D, Collins JP, et al. The Advisory Committee on Immunization Practices' interim recommendation for use of PfizerBioNTech COVID-19 vaccine in children aged 5-11 years-United States, November 2021. MMWR Morb Mortal Wkly Rep 2021;70:1579-83. PMID:34758012 https://doi.org/10.15585/mmwr. $\mathrm{mm} 7045 \mathrm{e} 1$

9. Food and Drug Administration. Pfizer-BioNTech COVID-19 vaccine EUA amendment review memorandum. Silver Spring, MD: US Department of Health and Human Services, Food and Drug Administration; 2021. https://www.fda.gov/media/148542/download

10. Malekifar P, Pakzad R, Shahbahrami R, et al. Viral coinfection among COVID-19 patient groups: an update systematic review and metaanalysis. BioMed Res Int 2021;2021:5313832. PMID:34485513 https:// doi.org/10.1155/2021/5313832 